\title{
Cigarette smoking has a positive and independent effect on testosterone levels
}

\author{
Wei Wang, ${ }^{1 *}$ Xiaobo Yang, ${ }^{2,3}$ Jianbo Liang, ${ }^{4}$ Ming Liao, ${ }^{2}$ Haiying Zhang, ${ }^{2,3}$ Xue Qin, ${ }^{2,5}$ \\ Linjian Mo, ${ }^{1,2}$ Wenxin Lv, ${ }^{2}$ Zengnan Mo ${ }^{1,2}$
}

${ }^{1}$ Department of Urology, the People's Hospital of Guangxi Zhuang Autonomous Region, ${ }^{2}$ Center for Genomic and Personalized Medicine, Guangxi Medical University, ${ }^{3}$ Department of Occupational Health and Environmental Health, School of Public Health of Guangxi Medical University, ${ }^{4}$ Department of Urology, the People's Hospital of Guangxi Zhuang Autonomous Region, ${ }^{5}$ Department of Clinical Laboratory, First Affiliated Hospital of Guangxi Medical University, Nanning, Guangxi Zhuang Autonomous Region, China

*Wei Wang and Xiaobo Yang have contributed equally to this work and should be considered as co-first authors.

\begin{abstract}
Previous studies have suggested that testosterone levels are linked to a variety of diseases, such as cardiovascular disease, type- 2 diabetes, the metabolic syndrome, erectile dysfunction, depression, stroke and osteoporosis. Since cigarette smoking is a major health problem and highly prevalent among men, several groups have studied the effects of cigarette smoking on testosterone levels in men. However, the results have been conflicting. Our objectives were to examine the association of cigarette smoking and serum levels of sex hormone-binding globulin (SHBG), total testosterone (TT) and free testosterone (FT) in a large male population. Data from 2,021 men (989 nonsmokers and 1,032 smokers), aged 20-69, were collected from the Fangchenggang Area Male Health and Examination survey using an in-person interview and self-administered questionnaires from September to December, 2009. We have found the following: (a) smokers had significantly higher TT and FT levels compared to nonsmokers, even after stratification as per age, BMI, triglycerides and alcohol consumption. (b) Both TT $(\mathrm{r}=\mathbf{- 0 . 0 8 3}, P<0.001)$ and $\mathrm{FT}(\mathrm{r}=\mathbf{- 0 . 2 7 1}, P<0.001)$ levels were negatively correlated to the amount of tobacco exposure. (c) Smoking was an independent influencing factor for the levels of both TT (unadjusted OR $=1.64,95 \%$ CI: 1.33-2.01, $P<0.001$; adjusted OR $=1.69,95 \%$ CI: 1.34-2.13, $P<0.001$ ) and FT (unadjusted $O R=1.32,95 \%$ CI: 1.08-1.61, $P=0.007$; adjusted $\mathrm{OR}=1.27,95 \% \mathrm{CI}$ : 1-1.61, $P=\mathbf{0 . 0 5 0}$ ) levels in multivariate logistic regression models before and after adjusting for age, BMI, fasting blood glucose, triglycerides, alcohol consumption and estradiol. (d) Smoking was not found to be an independent predictor of SHBG level after adjustment for confounders in multivariate regression model $(P>0.05)$, although a positive
\end{abstract}


association between increasing pack-years and SHBG level was observed $(r=0.174, P<0.001)$. More research is needed to elucidate the biological mechanisms and clinical significance of these associations.

Key words: Cigarette Smoking, Free testosterone, Sex hormone-binding globulin, Total testosterone

\section{INTRODUCTION}

The total testosterone (TT) levels measured and reported in most studies include SHBG-bound testosterone, albumin-bound testosterone and unbound or free testosterone (FT). Several studies have previously reported that testosterone levels are linked to a variety of conditions and diseases, such as cardiovascular disease, type-2 diabetes, the metabolic syndrome, erectile dysfunction, depression, obesity, stroke and osteoporosis. ${ }^{1-7}$ In addition, some studies have also demonstrated that men with low levels of free testosterone and bioavailable testosterone are at a higher risk of mortality, ${ }^{8-10}$ and that testosterone administration can increase the risk of cardiovascular adverse events as well. ${ }^{7}$ To reduce the risks of the abovementioned and other hormone-associated conditions, it is important that factors influencing hormone levels be identified.

Probable and possible associations of several factors, including age, cigarette smoking, body mass index (BMI), alcohol consumption and physical activity with hormone levels have been reported. ${ }^{11-17}$ It is believed that $\sim 34.8 \%$ of men worldwide are in the habit of smoking ${ }^{18}$ and there have been widespread concerns regarding the relationship between testosterone levels and smoking. However, the reported effects of smoking on testosterone levels in men are controversial, with some evidence even suggesting no association $^{15,19-24}$ and others reporting higher ${ }^{13,14,25-32}$ or lower ${ }^{33,34}$ levels of TT among smokers compared to nonsmokers. In these studies, we also found similarly conflicting reports with regard to correlations of smoking and FT (free testosterone) and sex hormonebinding globulin (SHBG). ${ }^{13,14,23,31}$ Such discrepancies may be attributed to differences among these studies such as study sample sizes, potential confounders used for adjustment and the methods employed for estimating tobacco exposure.
The aim of our study was to examine the relationship between smoking level (defined by pack-years, a common method used to estimate tobacco exposure) and levels of TT, FT and SHBG in 2,021 Chinese men who had participated in the Fangchenggang Area Male Healthy and Examination Survey (FAMHES) in 2009.

\section{MATERIAL AND METHODS}

\section{Study subjects}

The FAMHES is a population-based study conducted among non-institutionalized Chinese people in the age range of 17 to 88 years in Guangxi province. The study was designed to investigate the effects of environmental and genetic factors and their interaction with the development of age-related chronic diseases. A comprehensive demographic and health survey was conducted among 4,303 males who participated in the large-scale physical examination at the Fangchenggang First People's Hospital Medical Centre from September 2009 to December 2009. Following provision of written informed consent by the subjects, they were asked to answer standardized health questionnaires involving demographic characteristics (age, education, occupation, marital status), lifestyle characteristics (smoking, alcohol consumption and physical activity), health status, family history and medical histories (previous surgical procedures, ongoing medication and previous cardiovascular, endocrine, liver disease, urogenital tract diseases or neoplastic conditions). All data were collected through a face-to-face interview conducted by trained physicians. Upon completion of the interview, a $10 \mathrm{ml}$ sample of venous blood was obtained from each participant.

The current cross-sectional study was confined to 4,043 men aged 20 to 69 years old. Exclusion criteria for the study included the presence of self-reported diabetes mellitus (DM), neoplastic conditions, cir- 
rhosis or any other liver disease, infection with acute infectious diseases, use of any drug with a known effect on the endocrine system such as medication or other forms of hormonal therapy, and incomplete data with regard to smoking habits or consumption of alcohol on the previous day. Our final sample comprised 2,021 Chinese men.

Written informed consent was obtained from all subjects in the study. The research protocol was approved by the Ethics and Human Subject Committees of the First Affiliated Hospital of Guangxi Medical University.

\section{Anthropometric measures and blood samples}

Age, ${ }^{11,14-17}$ body mass index (BMI), ${ }^{12}$ fasting blood glucose,${ }^{1,35}$ triglycerides, ${ }^{36}$ alcohol consumption ${ }^{13,14}$ and estradiol levels, ${ }^{13}$ which have all been reported to be associated with alteration in hormone levels, were chosen as potential confounding factors in this study. Anthropometric measurements were performed by trained personnel using a standardized protocol. Body height and weight were measured with participants wearing light clothing and no shoes; body mass index (BMI) was calculated as weight in kilograms divided by the square of the height in meters $\left(\mathrm{kg} / \mathrm{m}^{2}\right)$. Men who had BMI $\geq 24$ were considered overweight ${ }^{37}$ and those with triglycerides $\geq 1.7 \mathrm{mmol} / \mathrm{l}$ were defined as having hypertriglyceridemia.

Overnight fasting venous blood specimens were drawn between 7:00 and 10:00 AM. All serum samples were stored at $-80^{\circ} \mathrm{C}$ until required for processing in the laboratory. Glucose, total cholesterol, triglycerides and albumin were measured enzymatically on a Dimension-RxL Chemistry Analyzer (Dade Behring, Newark, DE, USA) in the Department of the Clinical Laboratory at the Fangchenggang First People's Hosptal, using original reagents. Serum luteinizing hormone (LH), estradiol, SHBG and TT were measured by electrochemiluminescence immunoassay on Cobas 6000 system E601 (Elecsys module) immunoassay analyzers (Roche Diagnostics, IN, Germany) using reagents from the same batch. Intra-assay coefficients of variations were $3.6 \%$ for LH, 3.4\% for estradiol, $3.6 \%$ for TT and $4.4 \%$ for SHBG, respectively. FT values were calculated from TT and SHBG, based on the procedure described by Vermeulen et al (1999). ${ }^{38}$

\section{Quantification of smoking}

Self-report data on smoking habits such as number of cigarettes smoked per day and duration of smoking (measured in years) were collected during personal interviews. Participants were categorized as smokers and nonsmokers based on their smoking habits. Men who had smoked cigarettes for 6 months or longer and reported that they were still smoking at the time of the interview were considered "smokers", whereas men who had stopped smoking since more than 6 months prior to the interview as well as those who had never smoked were considered "nonsmokers". In addition, to take their entire history of smoking into account, pack-years of smoking were calculated by multiplying the number of packs smoked per day (1 pack $=20$ cigarettes) by the number of years smoked. ${ }^{39}$ These data were binned into the following quartiles: $0.05-5,5.01-11,11.01-20$ and $>20$ pack-years. It is believed that categorizing retrospectively calculated pack-years helps reduce potential misclassification biases. ${ }^{23,39}$ Moreover, in order to evaluate the effect of tobacco on hormones, smokers were also classified according to the number of cigarettes smoked per day $(1-10$ and $>10)$.

\section{Statistical analyses}

All statistical analyses were performed using SPSS 17.0. Results are expressed as mean \pm standard deviation (SD), unless otherwise stated. Normal distribution was evaluated by histograms and by determination of skewness. TT and FT appeared to be normally distributed; SHBG, LH and estradiol distributions were slightly skewed. Student's t-test was used to compare the means of normally distributed data and the Mann-Whitney U-test was used to compare medians of data that were not distributed normally. Two-way ANOVA was used to compare the means of TT, SHBG and FT values between nonsmokers and smokers, after stratifying all participants according to age, BMI, triglycerides and alcohol consumption. Analysis of covariance was utilized to compare the means of TT, SHBG and FT values for different groups classified based on pack-years of smoking or the number of cigarettes smoked per day. The Student-Newman-Keuls (SNK) test was used for the mutual comparison between multiple groups. The relationships between smoking and serum levels of 
TT, SHBG and FT were tested using a multivariate logistic regression model in which hormone levels were treated as the dependent variable. Odds ratio (OR) and $95 \%$ confidence intervals (CIs) were calculated for smokers who were in the highest quartile of the dependent variable as compared with nonsmokers, before and after adjusting for age, BMI, fasting blood glucose, triglycerides, alcohol consumption and estradiol levels. All statistical tests were two-tailed and statistical significance was defined as $\mathrm{P}<0.05$.

\section{RESULTS}

Characteristics of the study populations are shown in Table 1. Participants were in the age range of 20 to 69 years and the average age was $37.4 \pm 11.2$. Among the 2,021 volunteers recruited for the study, 1,032 subjects $(51.06 \%)$ reported themselves to be smokers and 989 (48.94\%) were nonsmokers. Smokers appeared to have significantly higher serum mean levels of triglycerides $(\mathrm{P}=0.002)$, estradiol $(\mathrm{P}=$ $0.023)$, TT $(\mathrm{P}<0.001)$ and FT $(\mathrm{P}<0.001)$ and lower levels of $\mathrm{LH}(\mathrm{P}=0.055$; borderline significant $)$ as compared with nonsmokers. No significant difference was observed in the mean values of SHBG and BMI between smokers and nonsmokers $(\mathrm{P}=0.169$ and
0.269 , respectively). Furthermore, only 72 men were considered to be "former smokers" in this study, and the exclusion of these samples from the group of nonsmokers did not affect the results (data not shown).

After considering the potential confounding factors for hormones and protein levels, we stratified all participants according to age, BMI, triglycerides and alcohol consumption. As indicated by the data in Table 2, smokers had significantly higher TT and FT levels in stratification analyses (all $P<0.05$ ). After stratification for age, smokers had significantly higher SHBG concentration $(P=0.023)$. However, no significant difference was found in the mean values of SHBG between smokers and nonsmokers after stratification for BMI, triglycerides and alcohol consumption $(P$ $>0.05$ ), although the SHBG level among smokers appeared to be higher than that of nonsmokers in every subgroup. Furthermore, the results also show that age, BMI and triglycerides were important and independent factors that influence sex hormones levels (all $P<0.01$ ).

To further evaluate the dose-response effect of cigarette smoking on SHBG, TT and FT levels, respectively, smokers were stratified into quartiles according to pack-years $(0.01-5,5.01-11,11.01-20$,

Table 1. Selected characteristics and hormone concentrations of 2,021 adult men who participated in the FAMHES study

\begin{tabular}{|c|c|c|c|c|}
\hline Characteristics & $\begin{array}{l}\text { All samples } \\
(\mathrm{N}=\mathbf{2 0 2 1})\end{array}$ & $\begin{array}{c}\text { Nonsmokers } \\
(\mathrm{N}=989)\end{array}$ & $\begin{array}{c}\text { Smokers } \\
(N=1032)\end{array}$ & $\mathbf{P}$ \\
\hline$\overline{\text { Age }}$ & $37.4 \pm 11.1$ & $38.2 \pm 12.1$ & $36.7 \pm 10.2$ & 0.002 \\
\hline BMI $\left(\mathrm{kg} / \mathrm{m}^{2}\right)$ & $23.2 \pm 3.3$ & $23.3 \pm 3.2$ & $23.2 \pm 3.4$ & 0.269 \\
\hline Alcohol consumption & $85.9 \%$ & $40.6 \%$ & $45.3 \%$ & $<0.001$ \\
\hline Glucose $(\mathrm{mmol} / \mathrm{l})$ & $5.3 \pm 1.0$ & $5.34 \pm 1.1$ & $5.3 \pm 1.0$ & 0.121 \\
\hline Total cholesterol $(\mathrm{mmol} / \mathrm{l})^{*}$ & $5.6(5.0 \sim 6.3)$ & $5.6(5.0 \sim 6.3)$ & $5.6(5.0 \sim 6.3)$ & 0.368 \\
\hline Triglycerides $(\mathrm{mg} / \mathrm{dl})^{*}$ & $99.2(68.2 \sim 154.5)$ & $93.9(65.5 \sim 147.0)$ & $102.7(70.0 \sim 164.7)$ & 0.002 \\
\hline Luteinizing hormone $(\mathrm{mIU} / \mathrm{ml})^{*}$ & $5.1(3.9 \sim 6.7)$ & $5.2(4.0 \sim 7.0)$ & $5.1(3.9 \sim 6.5)$ & 0.055 \\
\hline Estradiol $(\mathrm{pg} / \mathrm{ml})^{*}$ & $33.3(27.5 \sim 39.9)$ & $32.9(27.2 \sim 39.4)$ & $33.8(27.8 \sim 40.8)$ & 0.023 \\
\hline TT (nmol/l) & $21.6 \pm 6.6$ & $20.9 \pm 6.3$ & $22.3 \pm 6.7$ & $<0.001$ \\
\hline FT (ng/dl) & $12.1 \pm 3.6$ & $11.7 \pm 3.4$ & $12.5 \pm 3.7$ & $<0.001$ \\
\hline $\mathrm{SHBG}(\mathrm{nmol} / \mathrm{l})^{*}$ & $38.2(27.8 \sim 50.9)$ & $37.8(27.3 \sim 50.9)$ & $38.5(28.2 \sim 51.4)$ & 0.169 \\
\hline
\end{tabular}

Data are expressed as mean \pm standard deviation or as median (quartiles).

(Total cholesterol, Triglycerides) Smokers ( $\mathrm{N}=1015)$ Nonsmokers ( $\mathrm{N}=974)$; (Luteinizing hormone, Estradiol) Smokers $(\mathrm{N}=1008)$ Nonsmokers $(\mathrm{N}=967)$

Student's t-test was used to compare the means of normally distributed data.

* P-values were obtained in the Mann-Whitney U-test. 


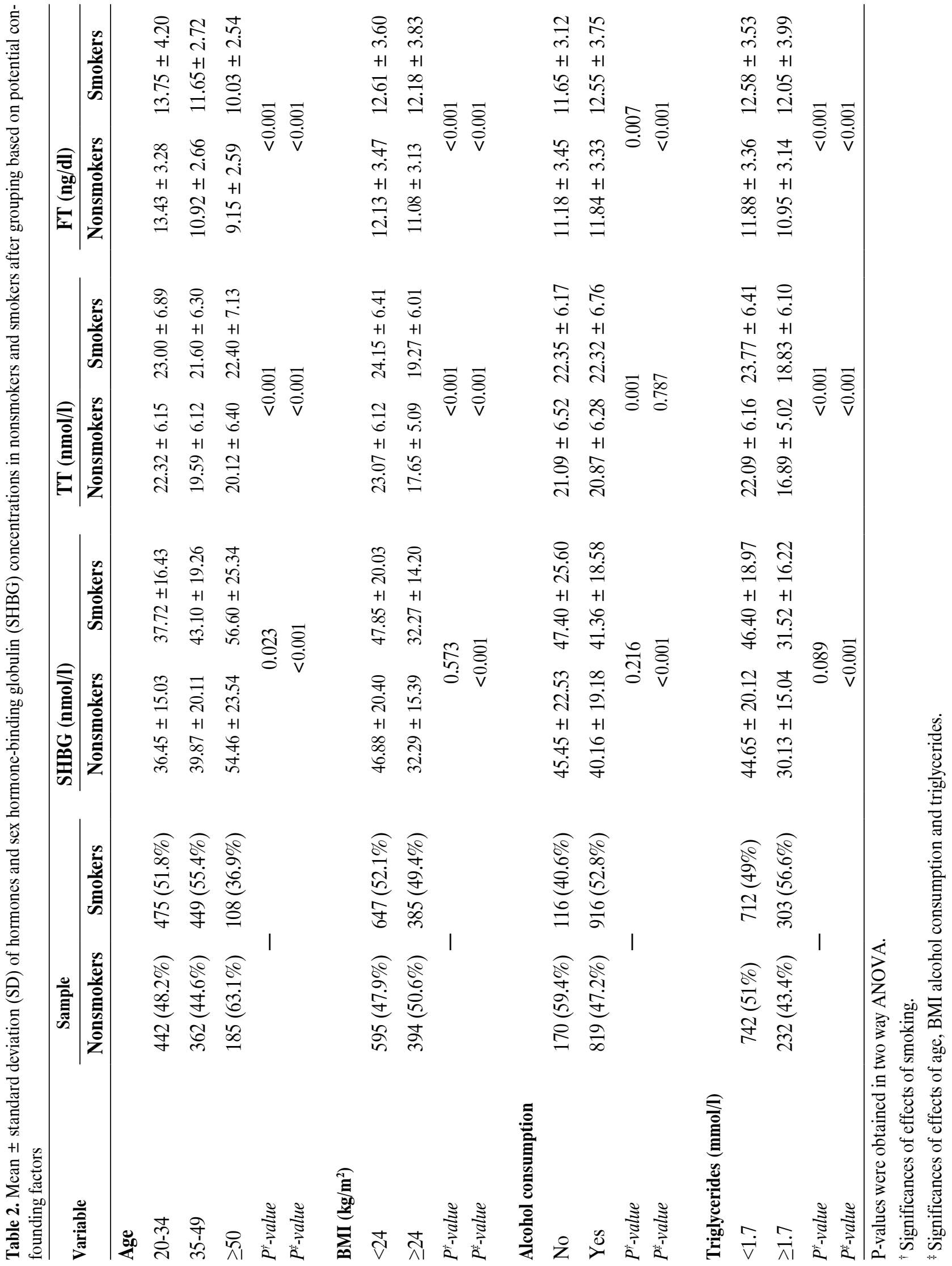


Table 3. Mean \pm standard deviation (SD) of hormones and sex hormone-binding globulin (SHBG) according to pack-years of smoking/the number of cigarettes smoked per day and comparison with nonsmokers in a sample of 2,021 men

\begin{tabular}{|c|c|c|c|c|c|c|c|}
\hline \multirow{2}{*}{$\begin{array}{l}\text { Tobacco exposure } \\
\text { Pack-years of smokir }\end{array}$} & \multirow[t]{2}{*}{$\mathbf{N}$} & \multicolumn{2}{|c|}{ SHBG (nmol/l) } & \multicolumn{2}{|c|}{ TT (nmol/l) } & \multicolumn{2}{|c|}{ FT (ng/dl) } \\
\hline & & & & & & & \\
\hline Nonsmokers & 989 & $41.07 \pm 19.90$ & Ref & $20.91 \pm 6.3$ & Ref & $11.71 \pm 3.4$ & Ref \\
\hline $0.01 \sim 5$ & 285 & $38.51 \pm 17.54$ & 0.381 & $23.48 \pm 6.70$ & 0.038 & $14.01 \pm 4.36$ & 0.028 \\
\hline $5.01 \sim 11$ & 245 & $41.10 \pm 19.07$ & 0.275 & $22.45 \pm 6.64$ & 0.005 & $12.68 \pm 3.51$ & 0.309 \\
\hline $11.01 \sim 20$ & 272 & $42.39 \pm 18.54$ & 0.423 & $21.71 \pm 6.70$ & 0.035 & $11.82 \pm 3.13$ & 0.264 \\
\hline$>20$ & 230 & $46.99 \pm 22.56$ & 0.133 & $21.51 \pm 6.57$ & 0.013 & $11.00 \pm 2.73$ & 0.284 \\
\hline$P$ & & 0.454 & & 0.007 & & 0.218 & \\
\hline \multicolumn{8}{|c|}{ Number of cigarettes/day } \\
\hline Nonsmokers & 989 & $41.07 \pm 19.90$ & Ref & $20.91 \pm 6.3$ & Ref & $11.71 \pm 3.4$ & Ref \\
\hline $1 \sim 10$ & 470 & $40.92 \pm 18.78$ & 0.407 & $22.51 \pm 6.70$ & 0.007 & $12.82 \pm 4.02$ & 0.078 \\
\hline$\geq 11$ & 562 & $42.97 \pm 20.19$ & 0.058 & $22.17 \pm 6.68$ & 0.001 & $12.13 \pm 3.37$ & 0.174 \\
\hline$P$ & & 0.149 & & 0.001 & & 0.154 & \\
\hline
\end{tabular}

$P$-values reflect significance levels for analysis of covariance after adjusting for age, BMI, fasting blood glucose, triglycerides, alcohol consumption and estradiol.

$>20$ ), or into two groups based on the number of cigarettes smoked per day $(1-10, \geq 11)$. In multivariable regression models, smokers had significantly higher TT levels in nearly all subgroups $(\mathrm{P}<0.05)$ as compared with nonsmokers, with the exception of the subgroup with 11.01-20 pack-years (Table $3)$. Only the least smoking subgroup (0.01-5 pack years) had a significantly higher level of FT than for nonsmokers. Our results also indicate that smokers had similar SHBG levels in each smoking subgroup as compared with nonsmokers, even after adjusting for confounding factors $(\mathrm{P}>0.05)$.

We also analyzed the association between the levels of hormones and SHBG and pack-years of smoking among smokers by calculating the Pearson correlation coefficient. Our results indicate that both TT and FT are significantly and negatively associated with smoking pack-years $(\mathrm{r}=-0.083, \mathrm{P}<0.001$; and $\mathrm{r}=-0.271, \mathrm{P}<0.001$, respectively). Additionally, serum SHBG level was found to be significantly and positively associated with smoking dose $(\mathrm{r}=0.174$, $\mathrm{P}<0.001)$.

Moreover, we classified subjects with the highest quartile of values as carriers of high hormone levels ( $\geqq 25.58 \mathrm{nmol} / \mathrm{l}$ for TT, $\geqq 14.05 \mathrm{ng} / \mathrm{dl}$ for FT and $>50.93 \mathrm{nmol} / \mathrm{l}$ for SHBG). Figure 1 further presents data indicating that smokers have a higher probability of circulating high levels of TT (unadjusted odds ratio $(\mathrm{OR})=1.64,95 \% \mathrm{CI}: 1.33-2.01, \mathrm{P}<0.001)$ and FT (unadjusted OR $=1.32,95 \%$ CI: $1.08-1.61, \mathrm{P}=$ $0.007)$ than nonsmokers in the unconditional logistic regression. After controlling for age, BMI, fasting blood glucose, triglycerides, alcohol consumption and estradiol levels, our data showed that smokers were still more likely to be in the highest quartile of TT (adjusted OR $=1.69,95 \%$ CI: 1.34-2.13, $\mathrm{P}<0.001$ ) and FT levels (adjusted OR $=1.27,95 \%$ CI: $1.00-1.61$, $\mathrm{P}=0.050$ ). The probability of possessing high SHBG levels was similar between smokers and nonsmokers before and after adjustment (unadjusted $\mathrm{OR}=1.04$, 95\% CI: 0.85-1.27, $\mathrm{P}=0.738$; adjusted $\mathrm{OR}=1.13$, 95\% CI: $0.90-1.42, \mathrm{P}=0.293$ ).

\section{DISCUSSION}

In this large-scale epidemiological study involving Chinese men, we found for the first time that smokers exhibit significantly higher levels of TT and FT when compared with age-matched nonsmokers. Our data support reports from several previous studies in other populations. Indeed, Corona et al (2005) analyzed 1,150 men in an outpatient clinic for sexual dysfunction and reported higher levels of TT in smokers, after 

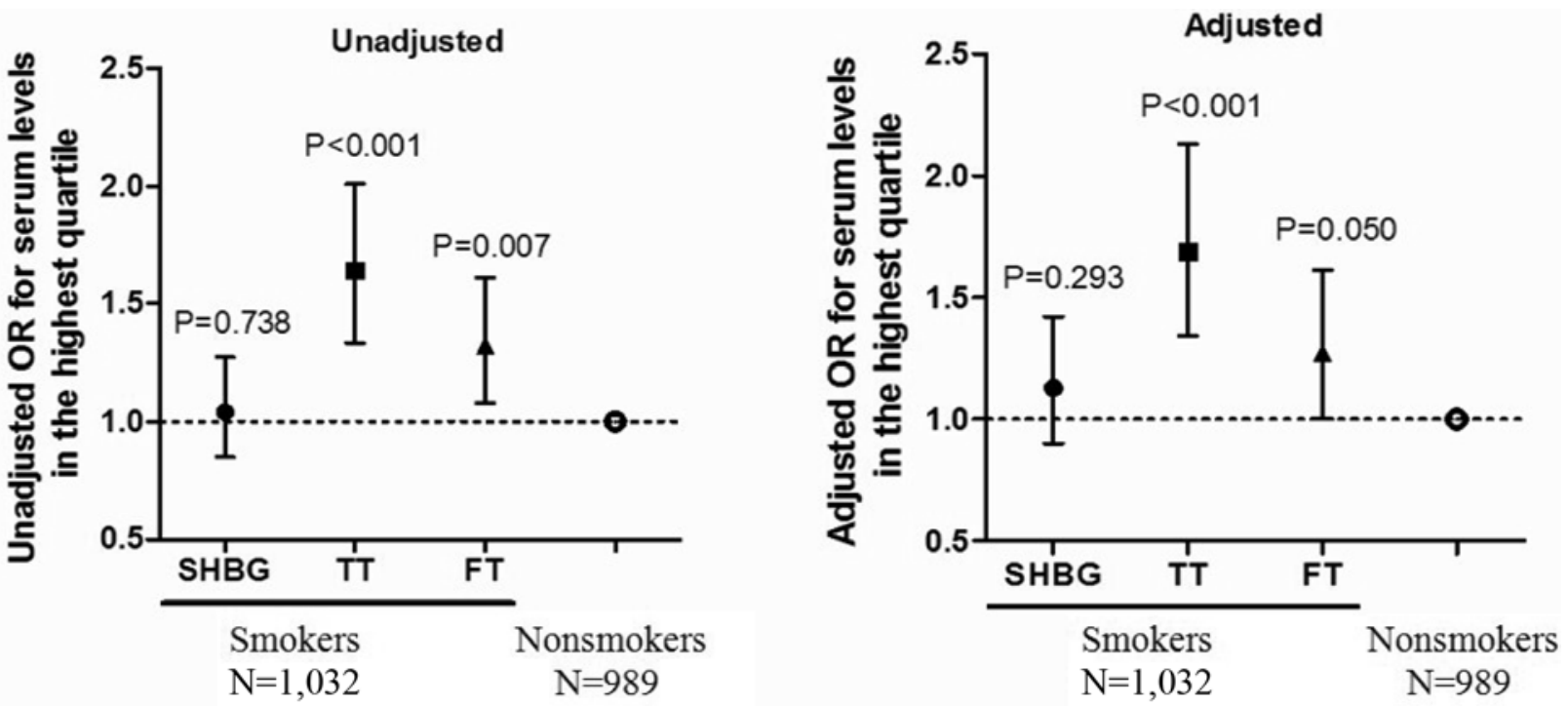

Figure 1. Unadjusted and adjusted odds ratios for hormonal serum levels in the highest quartile ( $\geqq 25.58 \mathrm{nmol} / \mathrm{l}$ for TT, $\geqq 14.05 \mathrm{ng} / \mathrm{dl}$ for FT, and $>50.93 \mathrm{nmol} / \mathrm{l}$ for SHBG) obtained in a multiple logistic regression containing the following factors: age, BMI, fasting blood glucose, triglycerides, alcohol consumption and estradiol levels. $\mathrm{OR}=$ odds ratio.

adjusting for age and BMI. ${ }^{32}$ Similarly, Svartberg et al (2003 and 2007) also reported that smokers exhibited higher TT levels. ${ }^{14,31}$ However, these reports were not corroborated by the findings of other studies..$^{15,19-24}$

Age, ${ }^{11,14-17} \mathrm{BMI}^{12}$ fasting blood glucose ${ }^{1,35}$ triglycerides ${ }^{36}$ alcohol consumption ${ }^{13,14}$ and estradiol levels ${ }^{13}$ have been proposed as potential predictors of testosterone levels in previous reports. Therefore, we not only treated them as co-variables in our multiple regression models, but also performed stratification analyses for SHBG, TT and FT by differences in age, BMI, triglycerides and alcohol-consumption subgroups. The results or trends were similar both prior to and after adjustment, which clearly suggests that, apart from age, BMI, triglycerides and alcohol-consumption, smoking is an important and independent factor that influences testosterone levels.

We also wondered whether any dose-response relationship existed between the extent of tobacco exposure and the levels of SHBG, TT and FT. Interestingly, our data showed that TT and FT levels gradually decreased with increasing tobacco exposure. This is the first large-scale study to show that the quantity of tobacco exposure in smoking is negatively associated with TT and FT levels among smokers, although smokers had higher levels of TT and FT than nonsmokers. However, previous studies have reported no such association or positive relationships. ${ }^{23,31}$ The study by Halmenschlager et al, comprising 255 men (2009) ${ }^{23}$ reported finding no clear associations between increased tobacco exposure and hormone and SHBG levels, which, however, could be partly attributed to their small sample size. Further, Svartberg et al (2007) ${ }^{31}$ analyzed 3,427 men in a cross-sectional population-based study and found that both TT and FT levels significantly increased with increasing tobacco exposure. However, these researchers employed number of cigarettes smoked per day, and not pack-years, which calculation takes into account a person's entire smoking history in order to quantify overall tobacco exposure.

No significant difference was observed in SHBG levels between smokers and nonsmokers in this study, which is consistent with a number of published reports..$^{13,20,23}$ On the other hand, several other studies have demonstrated higher SHBG levels in smokers. ${ }^{28,31,40}$ Since SHBG levels can be affected by a variety of conditions, the association seen in previous studies ${ }^{14,28,31}$ may be due to potential confounders, such as alcohol consumption and estradiol levels, which were adjusted for in our study but not in the abovementioned studies. Consistent with this explanation, the study by Shiels et al, in which percent body fat, 
alcohol intake and estradiol levels were considered as confounders and were adjusted for, also reported that smokers had smiliar SHBG levels to nonsmokers. ${ }^{13}$

The relationship between smoking and levels of TT, FT and SHBG is still elusive. English et al reported that smokers had higher SHBG levels than nonsmokers; however, no difference in bioavailable testosterone (BT) was observed. Since SHBG is the main carrier of testosterone (65-80\%), the increase in TT concentration may have been followed by increase in SHBG levels. ${ }^{28}$ However, there was no significant difference in SHBG levels between smokers and nonsmokers in our study, and similar results have been reported in other studies. ${ }^{13}$ Further, in agreement with previous studies, ${ }^{14,31}$ adjustment for SHBG in our study did not change the association between testosterone levels and smoking. Thus, the increase in TT levels may have been influenced more by increased BT levels. Consistent with this explanation, TT levels were observed to be associated with alteration in calculation of BT levels in our analyses (data not shown). Instead of calculating BT, which is criticized for its unreliability, it is recommended that BT be assayed using the ammonium sulfate precipitation method. ${ }^{41}$ Unfortunately, this method was not available for application in our study and has rarely been reported in other studies. Further research will be required to determine the effects of smoking on measurements of BT.

Smoking is positively and significantly associated with TT and FT levels in men in the FAMHES, even after adjusting for age, BMI, fasting blood glucose, triglycerides, alcohol consumption and estradiol level. The mechanism mediating the effect of smoking on testosterone production remains unclear. Several studies have reported that smoking may affect testosterone production not only by chronic mechanisms but also by acute ones. More specifically, Krsmanovic et al have demonstrated that smoking could stimulate acutely the release of gonadotropin-releasing hormone $(\mathrm{GnRH})$ and $\mathrm{LH}^{42,43}$ Moreover, it has also been suggested that smoking can increase testosterone levels by reducing the conversion of testosterone to estradiol. ${ }^{44,45}$ Additionally, the effects of smoking on testosterone levels have been found to be related to the personal smoking history, and TT and FT levels appeared to be inversely associated with pack-years of smoking in our study. Therefore, it is possible that the effect of smoking on testosterone levels is also mediated indirectly through other mechanisms. Yardimci et al studied the effects of long-term cigarette smoking on Leydig cells and found that cigarette smoking reduces testosterone biosynthesis. ${ }^{46}$ Their finding implied that smoking could lead to a gradual decrease in TT levels following long-term exposure, which may be related to the toxic effects of smoke on Leydig cells. ${ }^{46}$

Several studies have evaluated the effects of cigarette smoking on testosterone levels based on classifications of current, former and never smokers. ${ }^{13,29,30}$ We treated the men who had stopped smoking for more than 6 months prior to the interview as former smokers in the study. We found that former smokers had similar levels of SHBG, TT and FT as never smokers both prior to and after adjusting for age, BMI, fasting blood glucose, triglycerides, alcohol consumption and estradiol levels (data not shown). It appears that the effects of cigarette smoking on testosterone levels are reversed upon smoking cessation. This finding is consistent with several others reported in the literature previously. ${ }^{13}$ Because the sample size of former smokers was only 72 in the present study, we treated them as nonsmokers, as was done by Halmenschlager et al in their study. ${ }^{23}$

Our study has certain limitations. As the results of our data are from a cross-sectional analysis, unrecognized confounding factors that may potentially be associated with smoking, alcohol, BMI and hormone concentrations may have affected the final results, even though we employed stratification analysis and statistical adjustments. Moreover, we only classified alcohol consumption with "yes" or "no" answers and did not measure frequency or quantity of alcohol consumption. In addition, smoking status, alcohol consumption and the number of cigarettes smoked per day were all self-reported, which could have resulted in a potential misclassification bias. Further, the endocrine field is rapidly transitioning towards mass spectrometry based assays, which are well on their way to replacing direct testosterone immunoassays. Unfortunately, these methods were not avail- 
able to us. Finally, some nonsmokers may have also been exposed to passive smoking, but it is difficult to quantify or investigate this association.

In conclusion, our study reports that smoking is a positive and independent predictor of TT and FT levels. However, TT and FT levels appear negatively correlated with the dose of tobacco exposure among smokers. Smoking can lead to an acute increase in testosterone levels, but this can decrease with chronic exposure to tobacco by long-term smoking. Therefore, the effects of smoking on testosterone levels depend on personal smoking history. In addition, SHBG concentration increased gradually with increase in pack-years, although no significant differences in SHBG level were observed between nonsmokers and smokers. We suggest that studies should be conducted to evaluate how much of the deleterious effects of smoking on health are mediated by their effect on sex steroids.

\section{ACKNOWLEDGMENTS}

This study was partially supported by grants from the National Natural Science Foundation of China (30945204, 81060234), Key Program and University Talents Highland Innovation Team of Guangxi (2012012D003, GJR201147-09), Chairman Science and Technology Fund and Tackle Program of Guangxi (1116-03, GKG1298003-07-01), Guangxi Science Fund for Distinguished Young Scholars (2012GXNSFFA060009), Guangxi Provincial Department of Finance and Education (2009GJCJ150). The funders had no role in study design, data collection and analysis, decision to publish, or preparation of the manuscript.

We express our sincere thanks to the local research teams from Fangchenggang First People's Hospital, Fangchenggang, China, for their contribution to the survey. No potential conflicts of interest relevant to this article were reported.

\section{REFERENCES}

1. Rhoden EL, Ribeiro EP, Teloken C, Souto CA, 2005 Diabetes mellitus is associated with subnormal serum levels of free testosterone in men. BJU Int 96: 867-870.

2. Laaksonen DE, Niskanen L, Punnonen K, et al, 2003 Sex hormones, inflammation and the metabolic syndrome: a population-based study. Eur J Endocrinol 149: 601-608.

3. Shores MM, Sloan KL, Matsumoto AM, et al, 2004 Increased incidence of diagnosed depressive illness in hypogonadal older men. Arch Gen Psychiatry 61: 162-167.

4. Isidori AM, Giannetta E, Gianfrilli D, et al, 2005 Effects of testosterone on sexual function in men: results of a meta-analysis. Clin Endocrinol (Oxf) 63: 381-394.

5. Orwoll ES, Belknap JK, Klein RF, 2001 Gender specificity in the genetic determinants of peak bone mass. $J$ Bone Miner Res 16: 1962-1971.

6. Corona G, Monami M, Boddi V, et al, 2010 Low Testosterone is Associated with an Increased Risk of MACE Lethality in Subjects with Erectile Dysfunction .J Sex Med 7: 1557-1564.

7. Basaria S, Coviello AD, Travison TG, et al, 2010 Adverse Events Associated with Testosterone Administration. N Engl J Med 363: 109-122.

8. Menke A, Guallar E, Rohrmann S, et al, 2010 Sex steroid hormone concentrations and risk of death in US men. Am J Epidemiol 171: 583-592.

9. Laughlin GA, Barrett-Connor E, Bergstrom J, 2008 Low serum testosterone and mortality in older men. $\mathrm{J}$ Clin Endocrinol Metab 93: 68-75.

10. Tivesten A, Vandenput L, Labrie F, et al, 2009 Low serum testosterone and estradiol predict mortality in elderly men. J Clin Endocrinol Metab 94: 2482-2488.

11. Tajar A, Forti G, O’Neill TW, et al, 2010 Characteristics of secondary, primary, and compensated hypogonadism in aging men: evidence from the European Male Ageing Study. J Clin Endocrinol Metab 95: 1810-1818.

12. Derby CA, Zilber S, Brambilla D, Morales KH, McKinlay JB, 2006 Body mass index, waist circumference and waist to hip ratio and change in sex steroid hormones: the Massachusetts Male Ageing Study. Clin Endocrinol (Oxf) 65: 125-131.

13. Shiels MS, Rohrmann S, Menke A, et al, 2009 Association of cigarette smoking, alcohol consumption, and physical activity with sex steroid hormone levels in US men. Cancer Causes Control 20: 877-886.

14. Svartberg J, Midtby M, Bonaa KH, et al, 2003 The associations of age, lifestyle factors and chronic disease with testosterone in men: the Tromso Study. Eur J Endocrinol 149: 145-152.

15. Harman SM, Metter EJ, Tobin JD, Pearson J, Blackman MR, 2001 Longitudinal effects of aging on serum total and free testosterone levels in healthy men. Baltimore Longitudinal Study of Aging. J Clin Endocrinol Metab 86: 724-731.

16. Feldman HA, Longcope C, Derby CA, et al, 2002 Age trends in the level of serum testosterone and other hormones in middle-aged men: longitudinal results from 
the Massachusetts male aging study. J Clin Endocrinol Metab. 87: 589-598.

17. Liu PY, Beilin J, Meier C, et al, 2007 Age-related changes in serum testosterone and sex hormone binding globulin in Australian men: longitudinal analyses of two geographically separate regional cohorts. J Clin Endocrinol Metab 92: 3599-3603.

18. Anonymous, 2009 World health organization. World health statistics 2009. in Geneva: WHO Press, pp, 8393.

19. Barrett-Connor E, Khaw KT, 1987 Cigarette smoking and increased endogenous estrogen levels in men. Am J Epidemiol 126: 187-192.

20. Hautanen A, Manttari M, Kupari M, et al, 1993 Cigarette smoking is associated with elevated adrenal androgen response to adrenocorticotropin. J Steroid Biochem Mol Biol 46: 245-251.

21. Richthoff J, Elzanaty S, Rylander L, Hagmar L, Giwercman A, 2008 Association between tobacco exposure and reproductive parameters in adolescent males. Int $\mathbf{J}$ Androl 31: 31-39.

22. Saadat M, 2009 Serum levels of testosterone and gonadotrophins with respect to smoking status and genetic polymorphism of GSTT1. Mol Biol Rep 36: 1353-1356.

23. Halmenschlager G, Rossetto S, Lara GM, Rhoden EL, 2009 Evaluation of the effects of cigarette smoking on testosterone levels in adult men. J Sex Med 6: 17631772 .

24. Kirbas G, Abakay A, Topcu F, et al, 2007 Obstructive sleep apnoea, cigarette smoking and serum testosterone levels in a male sleep clinic cohort. J Int Med Res 35: 38-45.

25. Deslypere JP Vermeulen A, 1984 Leydig cell function in normal men: effect of age, life-style, residence, diet, and activity.J Clin Endocrinol Metab 59: 955-962.

26. Vogt HJ, Heller WD, Borelli S, 1986 Sperm quality of healthy smokers, ex-smokers, and never-smokers.Fertil Steril 45: 106-110.

27. Field AE, Colditz GA, Willett WC, Longcope C, McKinlay JB, 1994 The relation of smoking, age, relative weight, and dietary intake to serum adrenal steroids, sex hormones, and sex hormone-binding globulin in middle-aged men. J Clin Endocrinol Metab 79: 13101316.

28. English KM, Pugh PJ, Parry H, et al, 2001 Effect of cigarette smoking on levels of bioavailable testosterone in healthy men. Clin Sci (Lond) 100: 661-665.

29. Ponholzer A, Plas E, Schatzl G, et al, 2005 Relationship between testosterone serum levels and lifestyle in aging men. Aging Male 8: 190-193.

30. Suzuki R, Allen NE, Appleby PN, et al, 2009 Lifestyle factors and serum androgens among 636 middle aged men from seven countries in the European Prospective Investigation into Cancer and Nutrition (EPIC). Cancer Causes Control 20: 811-821.

31. Svartberg J, Jorde R 2007 Endogenous testosterone levels and smoking in men. The fifth Tromso study. Int J Androl 30: 137-143.

32. Corona G, Mannucci E, Petrone L, et al, 2005 Psychobiological correlates of smoking in patients with erectile dysfunction. Int J Impot Res 17: 527-534.

33. Briggs MH, 1973 Cigarette smoking and infertility in men. Med J Aust 1: 616-617.

34. Shaarawy M, Mahmoud KZ, 1982 Endocrine profile and semen characteristics in male smokers. Fertil Steril 38: 255-257.

35. Kapoor D, Aldred H, Clark S, Channer KS, Jones TH, 2007 Clinical and biochemical assessment of hypogonadism in men with type 2 diabetes: Correlations with bioavailable testosterone and visceral adiposity. Diabetes Care 30: 911-917.

36. Makinen JI, Perheentupa A, Irjala K, et al, 2008 Endogenous testosterone and serum lipids in middle-aged men. Atherosclerosis 197: 688-693.

37. Bei-Fan Z, 2002 Predictive values of body mass index and waist circumference for risk factors of certain related diseases in Chinese adults: study on optimal cut-off points of body mass index and waist circumference in Chinese adults.Asia Pac J Clin Nutr 11: Suppl 8: 685693.

38. Vermeulen A, Verdonck L, Kaufman JM,1999 A critical evaluation of simple methods for the estimation of free testosterone in serum. J Clin Endocrinol Metab 84: 3666-3672.

39. Bernaards CM, Twisk JW, Snel J, Van Mechelen W, Kemper HC, 2001 Is calculating pack-years retrospectively a valid method to estimate life-time tobacco smoking? A comparison between prospectively calculated pack-years and retrospectively calculated pack-years. Addiction 96: 1653-1661.

40. Wu FC, Tajar A, Pye SR, et al, 2008 Hypothalamicpituitary-testicular axis disruptions in older men are differentially linked to age and modifiable risk factors: the European Male Aging Study. J Clin Endocrinol Metab 93: 2737-2745.

41. Giton F, Fiet J, Guechot J, et al, 2006 Serum bioavailable testosterone: assayed or calculated? Clin Chem 52: 474-481.

42. Krsmanovic LZ, Mores N, Navarro CE, et al, 1998 Muscarinic regulation of intracellular signaling and neurosecretion in gonadotropin-releasing hormone neurons.Endocrinology 139: 4037-4043.

43. Mendelson JH, Sholar MB, Mutschler NH, et al, 2003 
Effects of intravenous cocaine and cigarette smoking on luteinizing hormone, testosterone, and prolactin in men.J Pharmacol Exp Ther 307: 339-348.

44. Barbieri RL, Gochberg J, Ryan KJ, 1986 Nicotine, cotinine, and anabasine inhibit aromatase in human trophoblast in vitro. J Clin Invest 77: 1727-1733.

45. Osawa Y, Tochigi B, Tochigi M, et al, 1990 Aromatase inhibitors in cigarette smoke, tobacco leaves and other plants. J Enzyme Inhib 4: 187-200.

46. Yardimci S, Atan A, Delibasi T, Sunguroglu K, Guven MC, 1997 Long-term effects of cigarette-smoke exposure on plasma testosterone, luteinizing hormone and follicle-stimulating hormone levels in male rats. $\mathrm{Br} \mathrm{J}$ Urol 79: 66-69. 